\title{
SELF-SIMILAR BEHAVIOR IN SEMICONDUCTOR SUPERLATTICES
}

\author{
JUAN C. CASTRO-PALACIO \\ Departamento de Física, Universidad de Pinar del Río \\ Martí 270, Esq. 27 de Noviembre \\ Pinar del Río, Cuba. CP-20100 \\ FRANCISCO R. VILLATORO \\ Departamento de Lenguajes y Ciencias de la Computación \\ Universidad de Málaga, E-29071 Málaga, Spain \\ OMEL MENDOZA-YERO \\ GROC UJI, Departament de Física \\ Universitat Jaume I, E12080 Castelló, Spain \\ LUISBERIS VELÁZQUEZ-ABAD \\ Departamento de Física, Universidad Católica del Norte \\ Av. Angamos 0610, Antofagasta, Chile \\ JUAN A. MONSORIU* \\ Centro de Tecnologías Físicas \\ Universitat Politècnica de València \\ E-46022 Valencia, Spain \\ *jmonsori@fis.upv.es
}

Received January 28, 2011

Revised October 17, 2011

Accepted October 28, 2011

\begin{abstract}
The scattering of particles in fractal superlattices has been analyzed by means of the transfer matrix method. The fractal superlattice consists of alternating layers of semiconductor materials
\end{abstract}

\footnotetext{
*Corresponding author.
} 
following the rule of a Cantor set. This problem can be represented by a model of quantum particles scattering in piecewise constant potential wells. Fractal properties of the reflection coefficient versus the particle energy curves are examined comparatively to the curves when using the corresponding periodic potentials. The degree of self-similarity of the output variables has been quantified by means of the correlation function.

Keywords: Polyadic Cantor Set; Transfer Matrix Method; Quantum Scattering.

\section{INTRODUCTION}

Nowadays, it is possible to deposit very thin alternating layers (of a hundred of atoms thick) of semiconductor materials with different energy band gaps over a substrate. This can be done, for example, by using techniques such as: molecular beam epitaxy or metalorganic chemical vapor deposition. ${ }^{1,2}$ The result is a heterostructure whose physical properties are ruled by quantum effects which play a major role as layers become thinner. A current application of these heteostructures is the "Quantum Superlattices". ${ }^{3}$ There are many derived applications of supperlattices, such as, the double well heterostructures in semiconductor diode lasers which are present in the majority of compact disc players, laser printers, fiber optic communication links ${ }^{4}$ and many other applications. ${ }^{1,2}$

As a first approximation to the modeling of semiconductor superlattices in the absence of an external electric field, a quasi-one-dimensional system of rectangular quantum wells separated by potential barriers can be considered. The solution for the scattering by multiple quantum wells can be obtained with the transfer matrix method ${ }^{5}$ involving the straightforward multiplication of $2 \times 2$ matrices. ${ }^{6-8}$ This method allows us to introduce a numerical procedure based on a piecewise constant approximation for a general potential, ${ }^{9}$ the analysis of defects on otherwise periodic potentials and even the consideration of more complicated forms, such as fractal potentials. ${ }^{10-12}$ This method uses an effective mass for the electrons which accounts for the external forces applied to the electron in the quantum well heteroestructure. Here, the electrons are not considered as free particles since they are under the influence of other atoms. Fractals constitute geometric objects that are homogeneous and self-similar. ${ }^{13}$ Beyond the fact that fractal structures are largely spread in nature and have been found to be very useful to describe and understand several phenomena, they have also been found on the grounds of multiple technologies.
The pioneer models of superlattices were crystalline and periodic. Nowadays, it is feasible to build nonperiodic superlattices that are based on amorphous semiconductors. ${ }^{14}$ A common example of a crystalline superlattice consists of several layers of gallium arsenide (GaAs) placed in between aluminium gallium arsenide $\left(\mathrm{Al}_{x} \mathrm{Ga}_{1-x} \mathrm{As}\right)$ ones (Axel and Terauchi ${ }^{15}$ ). A typical amorphous device can be made by alternating layers of amorphous germanium (a-Ge) and amorphous silicon (a-Si) deposited on a silicon substrate, as the polyadic Cantor superlattices, experimentally obtained by Järrendahl et al., ${ }^{16}$ in which each layer is $1.4 \mathrm{~nm}$ thick. Numerical results obtained by authors in Ref. 16 indicate that the neglect of imperfections, such as, small layer thickness fluctuations and interface mixing, mainly influence the peak intensity, but the peak positions are much less affected. Therefore, a good agreement between theoretical simulation and experiments can be achieved which encourages the possibility for the theoretical design of new microelectronic devices in practical applications. ${ }^{12}$

The scattering properties of triadic Cantor Set fractal potentials have been studied by numerically implementing a simple transfer matrix method and by a comparison with the equivalent periodic structure potential in Ref. 17. In this case, the segments were taken to be energy barriers. Also for this model, approximate analytical formulae were obtained for the tunneling curves in fractal superlattices of quantum wells and compared to numerical results showing good accuracy. ${ }^{18,19}$ These results are relevant for the development of bandpass energy filters for electrons, semiconductor solar cells and solid state radiation sources of high frequency $(\sim 1 \mathrm{THz})$.

In line with previous works, ${ }^{20}$ we compare here the scattering properties of particles by generalized periodic potentials and by fractal ones in the form of a generalized Cantor set (GCS) where segments represent wells. The consequences of this fractal 
property of the potential have been explored in the reflection coefficient. The degree of self-similarity of the output variables has been quantified by means of the correlation function.

The outline of the paper is the following. In Sec. 2 we formally present the statement of the problem, the features of the generalized fractal potential and the methodology for the solution. In Sec. 3 results and discussions are shown. We make special emphasis in the discussion of the self-similarity properties shown by the reflection coefficient in comparison to the case of using the equivalent periodic potential. Finally in Sec. 4 some conclusions are drawn.

\section{PRESENTATION OF THE PROBLEM}

\subsection{Transfer Matrix Method}

The scattering of particles in one-dimensional potentials is driven by the one-dimensional timeindependent Schrödinger equation ${ }^{21-23}$ :

$$
-\frac{\hbar^{2}}{2 m} \frac{\partial^{2} \psi(x)}{\partial x^{2}}+V(x) \psi(x)=E \psi(x),
$$

where $\psi(x), m$ and $E$ are the particle wave function, mass and energy, respectively; $\hbar$ is the Planck's constant and $V(x)$ is the quasi-periodic potential which can be represented by a piecewise constant function.

The wavefunction $\psi_{i}$ on the region where the potential constant value is $V_{i}$, is the addition of two plane waves, $\psi_{i}(x)=\psi_{i}^{+}(x)+\psi_{i}^{-}(x)$, given by

$$
\psi_{i}^{ \pm}(x)=A_{i}^{ \pm} e^{ \pm j k_{i} x}, \quad k_{i}=\frac{1}{\hbar} \sqrt{2 m\left(E-V_{i}\right)},
$$

where $j=\sqrt{-1}, k_{i}$ is the local particle momentum, and $A_{i}^{ \pm}$are integration constants to be determined by applying the standard boundary conditions at the interfaces between succesive wells. In the paper, a distribution of potential wells of the same depth is considered, so the potential $V_{i}=-V$ in the wells and $V_{i}=0$ outside them.

The solution of Eq. (1) for a distribution of $N$ constant potential wells is obtained by means of the transfer matrix method, ${ }^{7,9}$ yielding:

$$
\left(\begin{array}{c}
A_{0}^{+} \\
A_{0}^{-}
\end{array}\right)=M\left(\begin{array}{c}
A_{N+1}^{+} \\
A_{N+1}^{-}
\end{array}\right),
$$

with

$$
M=D_{0}^{-1}\left(\prod_{i=1}^{N} D_{i} P_{i}\left(d_{i}\right) D_{i}^{-1}\right) D_{N+1},
$$

where

$$
D_{i}=\left(\begin{array}{cc}
1 & 1 \\
k_{i} & -k_{i}
\end{array}\right), \quad P_{i}\left(d_{i}\right)=\left(\begin{array}{cc}
e^{j k_{i} d_{i}} & 0 \\
0 & e^{-j k_{i} d_{i}}
\end{array}\right),
$$

where $d_{i}$ is the width of the $i$ th potential well.

The reflection coefficient $R$ of the scattering of a quantum particle, incoming from the left, with the $N$-well potential is determined by the coefficients of the matrix $M$,

$$
\left(\begin{array}{c}
A_{0}^{+} \\
A_{0}^{-}
\end{array}\right)=\left(\begin{array}{ll}
M_{11} & M_{12} \\
M_{21} & M_{22}
\end{array}\right)\left(\begin{array}{c}
A_{N+1}^{+} \\
0
\end{array}\right),
$$

where terms $A_{0}^{ \pm}$represent the integration constants of the plane wave functions, $N$ identifies the $N$ th well, and no backward particle can be found on the right side of the potential, so $A_{N-1}^{+}=0$. The matrix $M$ is obtained by applying the standard boundary conditions at the interface, that is, the continuity of both the wave function and its derivative. Finally, the reflection coefficient is expressed by

$$
R=\frac{\left|A_{0}^{-}\right|^{2}}{\left|A_{0}^{+}\right|^{2}}=\frac{\left|M_{21}\right|^{2}}{\left|M_{11}\right|^{2}},
$$

since $k_{N+1}=k_{0}$.

\subsection{Generalized Cantor Set Potentials}

Figure 1 shows a schematic representation of the fractal Cantor set along with the corresponding periodic representation. The fractal Cantor set is obtained by means of an iterative algorithm. Let us comment first the case of the triadic Cantor set (upper right part of Fig. 1). The first step (stage

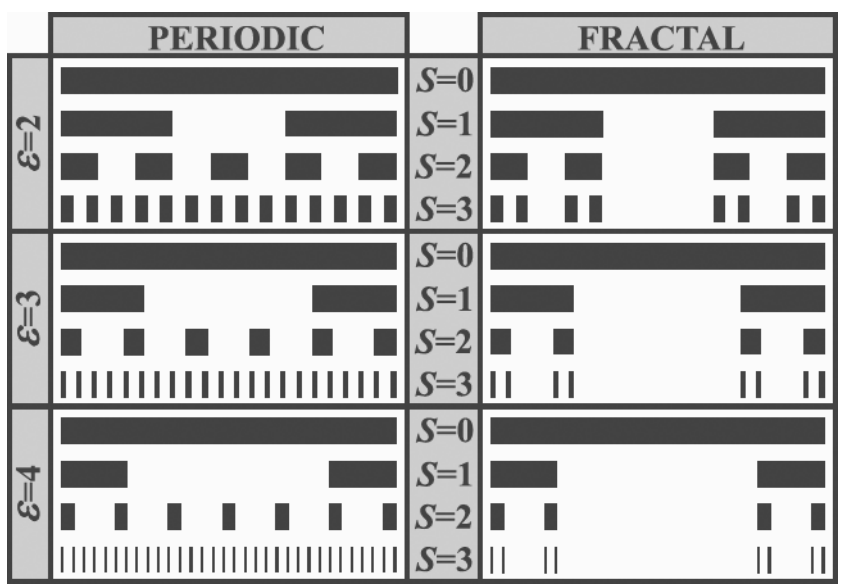

Fig. 1 Fractal Generalized Cantor Set and the corresponding finite periodic distributions. 
$S=0$ ) is to take a segment of unit length. The next one (stage $S=1$ ) is to divide the segment into three equal parts of length $1 / 3$ and remove the central one. In general, at the stage $S$, there are $2^{S}$ segments of length $3^{-S}$ with $2^{S}-1$ gaps in between. The stage $S+1$ is obtained by dividing each of these segments into three parts of length $3^{-S-1}$ and removing the central ones. The $S$ th stage Cantor set pre-fractal is interpreted as a quasi-periodic distribution of segments which can be obtained by removing some central segments in a finite periodic distribution. The periodic distribution at stage $S$ has $\left(3^{S}-1\right) / 2+1$ segments wells of length $3^{-S}$, separated by gaps of the same length, so that the period of this finite structure is $\Lambda=2 \times 3^{-S}$.

Following the above iterative process we can generalize the fractal Cantor set beyond the triadic one. The generalized structure consists of a sequence in which the ratio between lengths of the equivalent period $(\Lambda)$ and of the segment $(a)$ is a positive integer number $\varepsilon=\Lambda / a$. We call this parameter as generalization parameter of the Cantor set. In accordance with the above definition, a conventional (triadic) Cantor set ${ }^{20}$ is a particular case with $\varepsilon=2$. Thus the GCS can be obtained by dividing the segments into $(\varepsilon+1)$ parts and removing $(\varepsilon-1)$ central segments for each Cantor set characterized by the parameter $\varepsilon$. This operation will yield a family of generalized Cantor sets with equivalent periods: $\Lambda=\varepsilon(\varepsilon+1)^{-S}$.

The scattering problem for both the quasiperiodic Cantor sets and the finite periodic potential can be solved by the transfer matrix theory. ${ }^{17}$ In this work we consider quantum wells distributed in the black segments of the generalized distributions shown in Fig. 1. It is standard to normalize both the energy and the potential of the quantum wells by the length of the segment $a=(\varepsilon+1)^{S} L$, where $\varepsilon$ represents the generalized set, $S$ the stage in the set and $L$ is the unit segment at $S=0$. The resulting non-dimensional variables are:

$$
\phi=a \frac{\sqrt{2 m E}}{\hbar}, \quad \phi_{v}=a \frac{\sqrt{2 m V}}{\hbar} .
$$

\section{RESULTS AND DISCUSSIONS}

Figures 2-4 show the reflection coefficient, $R$, for the finite periodic potential and the GCS potential, respectively, in the region that includes the first band gap of the infinite periodic one. Three cases are represented, that is, for three values of the generalization parameter of the Cantor

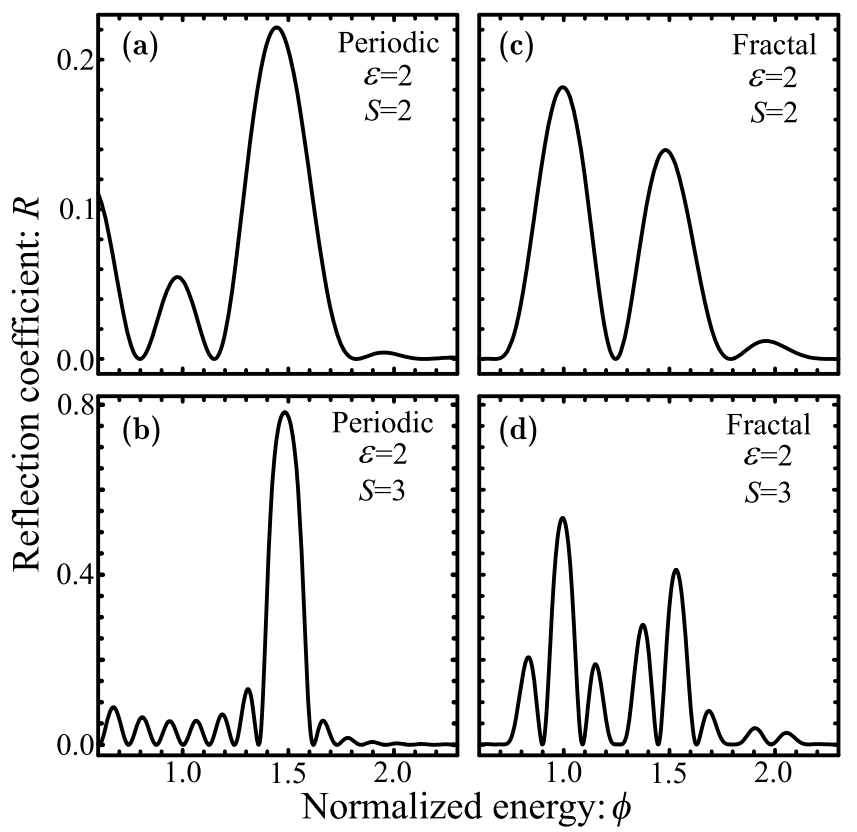

Fig. 2 Scattering reflection coefficient for the finite periodic and GCS potentials of stages $S=2$ and $S=3$ for the generalization parameter $\varepsilon=2$.

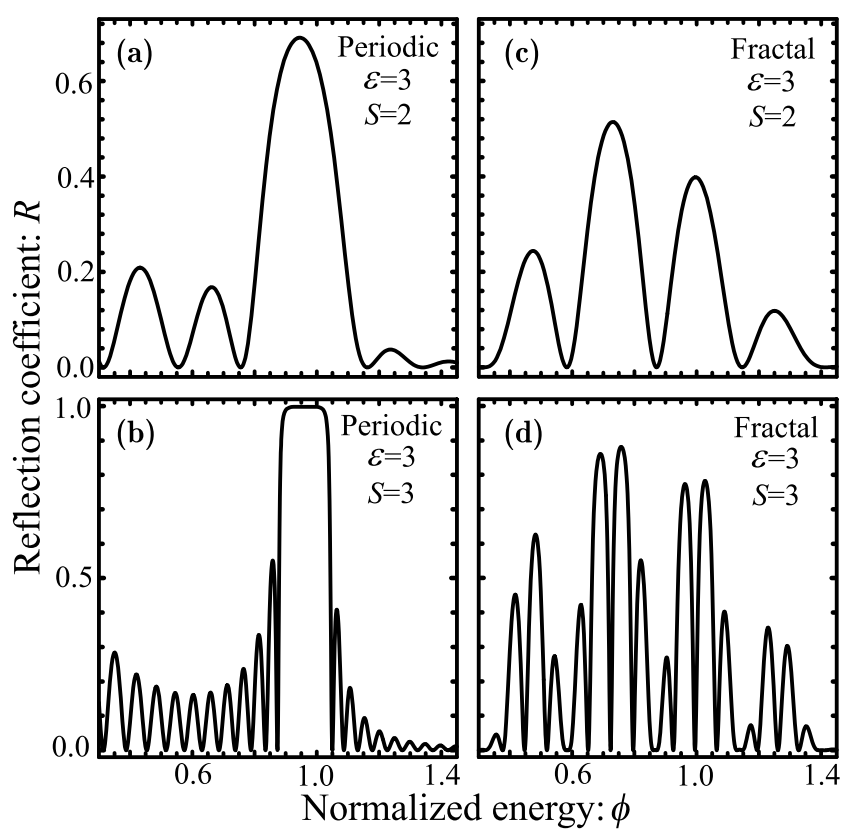

Fig. 3 Scattering reflection coefficient for the finite periodic and GCS potentials of stages $S=2$ and $S=3$ for the generalization parameter $\varepsilon=3$.

set: $\varepsilon=2,3$ and 4 . The potential well depth was set as $\phi_{v}=0.7$ for all calculations. Making use of the Kronig-Penney model, ${ }^{24}$ the band gap can be numerically calculated. The ranges are: $[1.437,1.542],[0.891,1.033]$ and $[0.613,0.778]$ for $\varepsilon=2,3$, and 4 , respectively. In this energy range, 


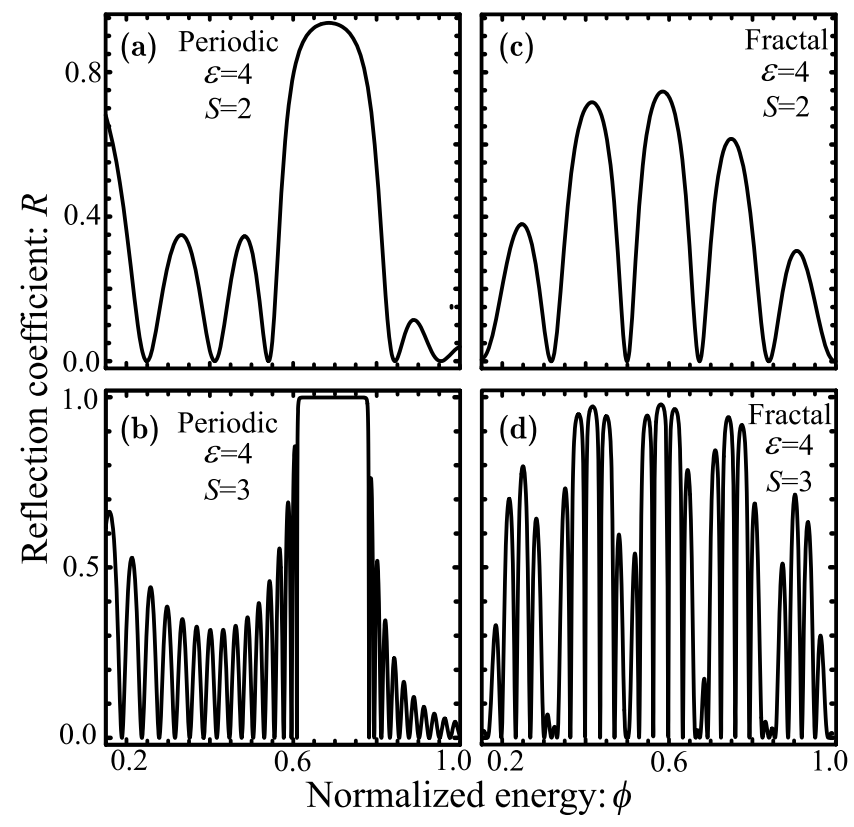

Fig. 4 Scattering reflection coefficient for the finite periodic and GCS potentials of stages $S=2$ and $S=3$ for the generalization parameter $\varepsilon=4$.

a Bloch wavefunction does not propagate in an infinite periodic potential and, therefore, the transmission coefficient ideally vanishes $(R=1)$; only evanescent wavefunctions characterized by a complex wavevector, $k$, are solution of the Schrödinger equation. For this reason, when the number of periods is finite, the quantum particle may pass through the potential distribution by the tunneling effect.

For finite periodic potentials (left panels in Figs. 2-4) it can be noticed that reflection coefficients approach unity as the number of periods in the spatial interval increase (going from $S=2$ to $S=3$ ), thus illustrating the process of appearance of the bandgap of the infinite periodic structure. The value of $R=1$ is never reached in the finite periodic structure.

In right panels of Figs. 2-4, the fractal cases for $S=2$ and $S=3$ are represented. It can be observed that the reflection at each higher stage is a modulated version of that associated with the previous stage. Namely, the reflection coefficient exhibits a characteristic fractal profile that reproduces the self-similarity of the potential distribution. For example, for each $\varepsilon$, any wide peak at the stage $S$ is transformed in $\varepsilon+1$ peaks at $S+1$. We can name this property as "Generalized self-similarity". It can also be seen that zero reflection occurs at specific discrete energies, while near total reflection is possible at other discrete energies. Moreover, an increasing number of zeros is observed in the fractal cases. These zeros represent resonances due to the presence of "defects" in the quasi periodic potential, obtained by removing some segments in the finite periodic sequence. In the limit $S \rightarrow 0$, the reflection coefficient for GCS fractal approaches unity with very narrow resonances in the interval shown in these figures. Even when the concept of fractal is strictly reached when $S$ goes to infinity, we have truncated the Cantor set at $S=3$ for each $\varepsilon$. Four terms are enough to see the self-similarity property of fractals as a tendency in the reflection coefficients.

In order to quantify the self-similarity of the reflection coefficient for the fractal potentials of Figs. 2-4, the correlation coefficient defined by Sakurada et $a l .{ }^{25}$ is shown in Fig. 5. A general function $f(x)$ is self-similar with respect to a point $x_{0}$ in its domain, $\left[x_{1}, x_{2}\right]$, if it remains unchanged when rescaling its domain with $x_{0}$ fixed, i.e., if $f\left(x_{0}+\left(x-x_{0}\right) / \gamma\right)=\gamma^{\alpha} f(x)$, where $\gamma$ is a scaling factor and $\alpha$ is the scaling exponent. Thus, the correlation function with respect to the point $x_{0}$ is defined by the following expression:

$$
C(\gamma)=\frac{\int_{x_{1}}^{x_{2}} f(x) f\left(x_{0}+\left(x-x_{0}\right) / \gamma\right) d x}{\sqrt{\int_{x_{1}}^{x_{2}} f^{2}(x) d x \int_{x_{1}}^{x_{2}} f^{2}\left(x_{0}+\left(x-x_{0}\right) / \gamma\right) d x}} .
$$

It can be noticed that $C(\gamma)=1$ for a self-similar function with respect to $x_{0}$. The curves in Fig. 5 indicate the correlation function of the reflection coefficient shown in Figs. 2-4 for the GCS potentials at the stage $S=3$. The local maxima at each curve are at $\gamma=\varepsilon+1$, that is, $\gamma=3$, 4, and

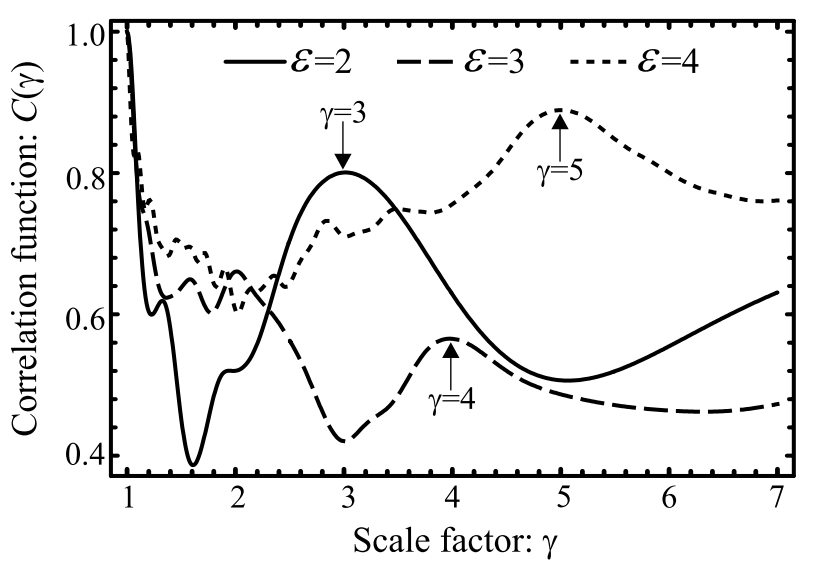

Fig. 5 Correlation function $\mathrm{C}(\gamma)$ for the reflection coefficients shown in fractal Figs. 2-4 for the stage $S=3$. 
$\gamma=5$ for $\varepsilon=2,3$, and 4 , respectively, clearly showing the self-similarity of the reflection coefficient as a consequence of the fractal structure of the potential.

\section{CONCLUSIONS}

The transfer matrix method has been used to solve the particle scattering at one-dimensional constant piecewise potentials in the form of a generalized fractal Cantor set which is a more complex new variant for the design of quantum superlattices. Results have been compared to the case when using the equivalent finite periodic potential. The selfsimilarity of the generalized Cantor fractal was well noticed in the curves of the reflection coefficients for each value of the generalization parameter $\varepsilon$ and was shown quantitatively by means of the correlation function. This results are particularly relevant for the design of diffractive lens applications.

One step beyond in this study can be the incorporation of disorders in both the finite periodic and fractal potentials by randomly perturbing the distance between the wells and the widths. This is a way to take into consideration the imperfections of small layer thickness fluctuations which will allow to arrive to more refined models. This work also suggests the use of the matrix transfer method to treat other interesting proposals of one-dimensional fractals such as Fibonacci sequence and Thue Morse sequences of potential wells or barriers with proper extensions to two-dimensional ones.

\section{ACKNOWLEDGMENTS}

We acknowledge the financial support from Ministerio de Ciencia y Tecnología (Grants DPI2008-02953 and TRA2009-0215), Spain. We also acknowledge the support from Generalitat Valenciana (PROMETEO2009-077 and ACOMP/2010/052) from Universitat Politècnica de València (PAID-0511), Spain.

\section{REFERENCES}

1. B. R. Nag, Physics of Quantum Well Devices (Kluwer Academic, Dordrecht, 2001).

2. P. Roblin, High-Speed Heteroestructure Devices: From Device Concepts to Circuit Modeling (Cambridge University Press, New York, 2002).

3. R. M. Kolbas and N. Holonyak, Jr., Man-made quantum wells: a new perspective on the finite square-well problem, Am. J. Phys. 52(5) (1984) 431-437.

4. P. S. Zory, Jr., Quantum Well Lasers (Academic Press, New York, 1993), pp. 1-13.

5. J. M. Ziman, Models of Disorder. The Theoretical Physics of Homogeneously Disordered Systems (Cambridge University Press, Cambridge, London, 1979).

6. M. Nawrocki and J. A. Gaj, Classical approach to the problem of an electron in a periodic potential, Am. J. Phys. 52 (1984) 807-809.

7. D. W. L. Sprung, H. Wu and J. Martorell, Scattering by a finite periodic potential, Am. J. Phys. 61 (1993) $1118-1124$.

8. T. P. Horikis, Eigenstate calculation of arbitrary quantum structures, Phys. Lett. A 359 (2006) 345348.

9. T. M. Kalotas and A. R. Lee, One-dimensional quantum interference, Eur. J. Phys. 12(6) (1991) 275282.

10. M. A. Herman and H. Sitter, Molecular Beam Epitaxy: Fundamentals and Current Status (Springer, Berlin, 1989).

11. E. L. Albuquerque and M. G. Cottam, Theory of elementary excitations in quasiperiodic structures, Phys. Rep. 376(4) (2003) 225-337.

12. E. Maciá, The role of a periodic order in science and technology, Rep. Prog. Phys. 69(2) (2006) 397-441.

13. A. J. Hurd, Resource Letter FR-1: Fractals, Am. J. Phys. 56(11) (1988) 969-975.

14. R. Mazurczyk, Semiconductor superlattices, Chaos Solitons Fractals 10(12) (1999) 1971-1982.

15. F. Axel and H. Terauchi, High-resolution xray-diffraction spectra of Thue-Morse GaAs-AlAs heterostructures: towards a novel description of disorder, Phys. Rev. Lett. 66 (1991) 2223-2226.

16. K. Järrendahl, M. Dulea, J. Birch and J. E. Sundgren, X-ray diffraction from amorphous Ge/Si Cantor superlattices, Phys. Rev. B 51 (1995) 7621-7631.

17. J. A. Monsoriu, F. R. Villatoro, M. J. Marín, J. F. Urchueguía and P. Fernández de Córdoba, A transfer matrix method for the analysis of fractal quantum potentials, Eur. J. Phys. 26(4) (2005) 603-610.

18. J. A. Monsoriu, F. R. Villatoro, M. J. Marín, J. Pérez and L. Monreal, Quantum fractal superlattices, Am. J. Phys. 74(9) (2006) 603-610.

19. F. R. Villatoro and J. A. Monsoriu, Tunneling in quantum superlattices with variable lacunarity, Phys. Lett. A 372(21) (2008) 3801-3807.

20. O. Mendoza-Yero, M. Fernández-Alonso, G. Mínguez-Vega, J. Lancis, V. Climent and J. A. Monsoriu, Fractal generalized zone plates, J. Opt. Soc. Am. A 26(5) (2009) 1161-1166.

21. C. Kittel, Introduction to Solid State Physics (Wiley, New York, 1996). 
22. N. W. Ashcroft and N. D. Mermin, Solid State Physics (Saunders, Philadelphia, 1976).

23. W. D. Heiss and W.-H. Steeb, Energy levels for a square well containing $\delta$-function barriers on a Cantor set, Phys. Rev. A 39 (1989) 6018-6021.

24. B. Schulkin, L. Sztancsik and J. F. Federici, Analytical solution for photonic band-gap crystals using
Drude conductivity, Am. J. Phys. 72(8) (2004) 1051-1054.

25. Y. Sakurada, J. Uozumi and T. Asakura, Fresnel diffraction by 1-D regular fractals, Pure Appl. Opt. 1(1) (1992) 29-40. 